\title{
Prevalence and predictors of anaemia among patients presenting with kidney diseases at the University of Dodoma Hospital in central Tanzania
}

\author{
ALFRED J. MEREMO 1,2 , MASUMBUKO Y. MWASHAMBWA ${ }^{1,2}$, MATOBOGOLO B. MASALU ${ }^{1,2}$, JANET KAPINGA ${ }^{2}$, \\ REHEMA TAGALILE ${ }^{2}$, DAVID P. NGILANGWA3 and ISSA SABI ${ }^{*}$ \\ ${ }^{1}$ School of Medicine \& Dentistry, College of Health Sciences, University of Dodoma, P.O Box 395, Dodoma, Tanzania \\ ${ }^{2}$ Haemodialysis Unit, University of Dodoma, P.O Box 259, Dodoma, Tanzania \\ ${ }^{3}$ Amref Health Africa, P.O Box 2773 Dar es Salaam, Tanzania \\ ${ }^{4}$ National Institute for Medical Research - Mbeya Medical Research Centre, P.O Box 2410 Mbeya, Tanzania
}

\begin{abstract}
Background: Anaemia is a common complication that contributes to the burden of kidney diseases. Anaemia confers significant risk of cardiovascular disease and contributes to decreased quality of life. While the primary cause of anaemia is the inadequate production of erythropoietin by the kidneys to support erythropoiesis, other factors may contribute to anaemia. The aim of this study was to determine the prevalence and predictors of anaemia among patients presenting with kidney diseases at the haemodialysis unit of the University of Dodoma (UDOM) hospital in central Tanzania.

Methodology: In this retrospective study we reviewed data of patients who presented at UDOM haemodialysis unit in Tanzania with kidney diseases as from January 2013 to June 2015. Data were descriptively and inferentially analysed using Stata version 11 software.

Results: A total of 1,395 patients were involved in this study. Of these, 792 (56.8\%) presented with kidney diseases, 249 (31.4\%) were found to have anaemia. The leading cause of anaemia was chronic kidney disease (CKD) 136 (54.6\%), blood loss 74(29.7), haemolysis 15 (6.0\%), Nutrition 13(5.2\%) and others 11 (4.4\%). Glomerular filtration rate of $<60 \mathrm{~mL} / \mathrm{min} / 1.73 \mathrm{~m}^{2}$ accounted for 59.1\% of CKD. Median [IQR] serum creatinine level: 246 [177 - 317] $\mu \mathrm{mol} / \mathrm{L}$, Urea level $16[8-24] \mathrm{mmol} / \mathrm{L}$ and haemoglobin of $9.8[6.2-13.4] \mathrm{g} / \mathrm{dL}$. Prevalence of anaemia was strongly associated with declining glomerular filtration rate $(P=0.01)$.

Conclusion: Anaemia is very common among patients presenting with kidney diseases. These patients require a thorough evaluation to identify and correct causes of anaemia other than erythropoietin deficiency.

Keywords: chronic kidney disease, prevalence, predictors, anaemia, Tanzania
\end{abstract}

\section{Introduction}

Anaemia is defined as a state in which the quality and/or quantity of circulating red blood cells is below normal or the established cut off defined by the World Health Organization (WHO, 2001). Anaemia begins to develop early in the course of chronic kidney disease (CKD). Lower levels of kidney functions have been associated with lower haemoglobin levels and a higher prevalence and severity of anaemia (Coresh et al., 2003). CKD has been defined as evidence of kidney damage based on abnormal urinalysis results or structural abnormalities observed on ultrasound images or a glomerular filtration rate (GFR) of less than $60 \mathrm{~mL} / \mathrm{min}$ for 3 or more months (NKF, 2006). The global CKD prevalence is estimated to be between 11 and 13\% (Hill et al., 2016). Future research should evaluate intervention strategies deliverable at scale to delay the progression of CKD and improve Cardiovascular Disease (CVD) outcomes.

Anaemia is a common complication that contributes to the burden of kidney diseases. High prevalence of anaemia among CKD patients has been reported in the United Kingdom (NCGC, 2015), Singapore (Lau et al., 2015) and United States (Stauffer et al., 2014).

*Correspondence E-mail: isabi@nimr-mmrc.org 
While the primary cause of anaemia is the inadequate production of erythropoietin by the kidneys to support erythropoiesis (Ramanath et al., 2012), other factors may contribute to anaemia (McFarlane et al., 2008; Hsu et al., 2002). These include iron-deficiency, recent bleeding (WHO, 2001), vitamin B12 or folate deficiency, myelodysplastic syndrome, acute leukaemia, chronic leukaemia and lymphoma-related disorders and multiple myeloma (NCGC, 2015). It has been known for some years that anaemia exists in patients with diabetes and CKD, and that this anaemia occurs early in the course of diabetic kidney disease and is associated with inappropriately low erythropoietin concentrations (Ishimura et al., 1998; Thomas et al., 2005; New et al., 2008; Mehdi et al., 2009). Several retrospective studies have reported a higher prevalence of anaemia when inflammatory processes, malnutrition or diabetes are present in severe CKD (Otero et al., 2010; Meuwese et al., 2011). In a study in Singapore, the probability of developing anaemia was greater for patients with stage 5 CKD and among those with haematological disorders and with respiratory disorders (Lau et al., 2015).

Anaemia causes significant risk of CVD and contributes to decreased quality of life (McClellan et al., 2004). Anaemia in CKD is associated with memory loss, disturbances in sleep, CKD progression, CVD co-morbidities and higher mortality rates (Herzog et al, 2009). Direct costs for health care are higher for CKD patients with anaemia when compared to those without anaemia (van Nooten et al, 2010). The quality of life issues (e.g. fatigue, depression, reduced productivity) are more common in patients with anaemia (Smith et al., 2010). In a study in South Africa, primary hypertension was reported to occur in a quarter and was the putative cause of End Stage Renal Disease (ESRD) among a larger proportion of the patients (Meyers, 2015). In northern Tanzania, among those with CKD, 19.3\% had hypertension alone, $14.0 \%$ had diabetes and hypertension, $7.0 \%$ had diabetes alone, $7.0 \%$ had HIV alone, and 3.5\% had HIV and hypertension (Stanifer et al., 2015). In another study northwestern Tanzania, it was reported that hypertension related diseases were the most common cause of hospital admissions and accounted for most number of deaths (Peck et al., et al., 2013).

Since anaemia is common among patients with kidney diseases and is associated with high mortality, most studies have concentrated on anaemia among patients who have CKD. The aim of this study is to determine the prevalence and predictors of anaemia among patients presenting with kidney diseases at the University of Dodoma haemodialysis unit. Improved clinical and haematology services as well as reassessment of empirical treatment guidelines for anaemia might contribute to better outcomes among patients who present with kidney diseases.

\section{Materials and Methods}

\section{Study design, population and settings}

This was a retrospective study that involved patients who presented with kidney diseases at the University of Dodoma (UDOM) Hospital Haemodialysis Unit in central Tanzania. The hospital has a bed capacity of 100 beds. The Haemodialysis Unit was established in January 2013 to serve as a referral centre for Singida, Morogoro, Iringa, Manyara, Tabora regional referral hospitals with a total population of 20 million people.

\section{Data collection and Laboratory procedures}

All patients with kidney diseases who presented at the Haemodialysis Unit from January 2013 to June 2015 were included. Data were carefully reviewed and all patients with incomplete records were excluded. The data included personal, clinical and laboratory findings. Information about age, sex, marital status, clinical signs (oedema, anuria, hypertension, Body mass Index), urinalysis, Hepatitis panel, full blood picture, urea, creatinine, electrolytes, random blood glucose and HIV test were 
carefully recorded. The estimated Glomerular Filtration Rate (eGFR) was calculated using the Chronic Kidney Disease Epidemiology Collaboration (CKD-EPI) equation. Bedside isotope-dilution mass spectrometry (IDMS) traceable Schwartz GFR Calculator for Children was used to stage patients. Outcome measures were anaemia, kidney diseases and death.

\section{Data analysis}

Data collected were entered into a computer using epidata version 3.1 (CDC, Atlanta, USA) and analysed using STATA version 11 (College Station, Texas, USA). Depending on variable distribution, either mean with standard deviation or median with interquartile range were used to summarize continuous data. The correlation between the development of anaemia and different patient parameters was determined by performing logistic regression analyses. Odds ratios (OR) were calculated to estimate the percentage change in risk of anaemia development. Parameters with $\mathrm{p}$ values $<0.05$ were considered statistically significant.

\section{Ethical considerations}

The study was approved by the University of Dodoma Research and Publications Ethics Review Board.

\section{Results}

\section{Demographic and other characteristics of the study population}

A total of 1,395 patients presented at The University of Dodoma haemodialysis unit. Out of all patients 151 (10.8\%) were excluded from this study due to incomplete records and 452 (32.4\%) patients had no kidney disease after screening. A total of 792 (56.8\%) patients were found to have kidney diseases and $383(48.3 \%)$ of them were males. The median age was 42 years (IQR $9-88$ ). (Table 1)

Table 1: Demographic characteristics of the patients $(\mathrm{N}=792)$

\begin{tabular}{lll}
\hline Characteristic & Response & Proportion or Median [IQR) \\
\hline Sex & Male & $383(48.3 \%)$ \\
& Female & $409(51.7 \%)$ \\
Age (years) & $42[9-88]$ & \\
Marital status & Single & $244(30.8 \%)$ \\
& Married & $356(45.0 \%)$ \\
& Divorced & $92(11.6 \%)$ \\
& Widow & $100(12.6 \%)$ \\
\hline
\end{tabular}

Thirty-eight (4.8\%) and 17 (2.1\%) patients had HIV and Hepatitis B infections, respectively. Glomerular filtration rate of $<60 \mathrm{~mL} / \mathrm{min} / 1.73 \mathrm{~m}^{2}$ accounted for $59.1 \%$ of CKD patients. Median [IQR] serum creatinine level: 246 [177 - 317] $\mu \mathrm{mol} / \mathrm{L}$, Urea level $16[8-24] \mathrm{mmol} / \mathrm{L}$ and haemoglobin of 9.8 [6.2 13.4] g/dL.

\section{Prevalence of anaemia}

Of the 792 patients 249 (31.4\%) were found to have anaemia. CKD was the leading cause of anaemia accounting for 136 (54.6\%) patients. Other causes included blood loss with 74 (29.7\%) patients, haemolysis with $15(6.0 \%)$ patients and nutrition with $13(5.2 \%)$ patients. Eleven (4.4\%) patients had anaemia attributed to infections and iatrogenic causes. Majority of the patients who presented with 
anaemia had hypertension (25.7\%) as the leading medical condition, followed by haematological disorders (23.3\%), gastrointestinal disorders (17.7\%) and diabetes mellitus (14.6\%) (Table 2).

Table 2: Medical conditions patients who presented with anaemia $(\mathrm{N}=249)$

\begin{tabular}{|c|c|}
\hline Medical condition & Anaemic patients \\
\hline & Percentage \\
\hline Hypertension & $64(25.7 \%)$ \\
\hline Haematological disorders & $58(23.3 \%)$ \\
\hline Gastrointestinal disorders & $44(17.7 \%)$ \\
\hline Diabetes mellitus & $36(14.6 \%)$ \\
\hline Heart failure & $24(9.6 \%)$ \\
\hline Hypertension and Diabetes & $12(4.8 \%)$ \\
\hline Others & $11(4 \cdot 4 \%)$ \\
\hline
\end{tabular}

\section{Predictors of anaemia}

A total of 15 predictors of anaemia among patients with kidney disease were identified after performing univariate logistic regression analyses. Backward elimination reduced this to 5 parameters. The potential predictors identified were sex, previous haemoglobin level, CKD stage, haematological disorders and heart failure. Sex and heart failure were found to be significant variables in univariate logistic regression analyses. However, this significance was lost after multivariate logistic regression analyses (Table 3).

Table 3: Adjusted odds ratios of developing anaemia (serum $\mathrm{Hb}<10 \mathrm{~g} / \mathrm{dL}$ )

\begin{tabular}{llcr}
\hline Parameters & & Adjusted OR (95\% CI) & P-value \\
\hline Sex & Male & 1.00 & \\
& Female & $0.80(0.33-1.76)$ & 0.678 \\
\hline CKD Stage & Stage 1 \& & 1.00 & \\
& Stage 3 & $0.78(0.12-3.19)$ & 0.882 \\
& Stage 4 & $1.57(0.31-5.75)$ & 0.362 \\
& ESRD & $15.65(2.38-69.42)$ & $<0.01^{*}$ \\
\hline Previous haemoglobin & & $0.43(0.34-0.55)$ & $<0.01^{*}$ \\
\hline Blood disorders & Blood disorders & $14.61(6.22-39.16)$ & $<0.01^{*}$ \\
Heart failure & Heart failure & $0.65(0.42-1.62)$ & 0.483 \\
\hline
\end{tabular}

Key: $\mathrm{Cl}=$ confidence interval; ${ }^{*} \mathrm{P}<0.05$

Previous haemoglobin level was predictive of developing anaemia (OR 0.43, $95 \% \mathrm{Cl}$ 0.34-0.55, $\mathrm{p}$ <0.01). In addition, the multivariate adjusted odds of developing anaemia were 15.6 times higher for a patient with ESRD (OR 15.65, 95 \% Cl 2.38- 69.42, p < 0.01). Patients with blood disorders (OR 14.61, $95 \% \mathrm{Cl} 6.22-39.16, \mathrm{p}<0.01)$ also had higher odds of developing anaemia. A total of 23 (9.2\%) patients among those who presented with anaemia died during the study period. Most of these patients presented with low haemoglobin levels, ESRD and disorders of the blood. 


\section{Discussion}

In this study, anemia was present in about one-third of the 792 patients with kidney disease and CKD was the leading cause of anaemia. Similar findings have been reported from other studies in the UK (27.5\%) (NCGC, 2015) and Singapore (35.4\%) (Lau et al., 2015). Lower prevalence has been reported from studies in the United States (Stauffer et al., 2014). The different findings could be due to differences in geographical location, lifestyle, racial and genetic make-up. Also could be accounted by the differences in the study populations.

The most common cause of anaemia was CKD. Similar findings have been reported from other studies (Lau et al., 2015; Ramanath et al., 2012; Mehdi et al., 2009; New et al., 2008; Thomas et al., 2005). The leading cause of CKD was hypertension which was as well the leading medical condition among patients who presented with anaemia. Similar findings have been reported in other studies in Tanzania and South Africa (Peck et al., 2013; Stanifer et al., 2015; Meyers, 2015). Different findings have been reported in the UK and USA whereby diabetes mellitus was found to be the leading cause of CKD and eventually anaemia (Coresh et al., 2003; Stauffer et al., 2014; NCGC, 2015). Slightly less than half of the patients who presented with anaemia had blood disorders, gastrointestinal disorders and other medical conditions apart from CKD causing their anaemia. Similar findings have been reported from other studies (Hsu et al., 2002; McFarlane et al., 2008).

Development of anaemia was higher for patients with ESRD. Similar findings have been reported from other studies (Ishimura et al., 1998; Thomas et al., 2005; New et al., 2008; Mehdi \& Toto, 2009). Previous haemoglobin level was predictive of developing anaemia and patients with blood disorders had higher chances of developing anaemia. Other studies have similarly reported higher prevalence of anaemia when inflammatory processes, malnutrition or diabetes mellitus were present in patients with kidney diseases (Otero et al., 2010; Meuwese et al., 2011). Although in our study respiratory disorders did not predict the development of anaemia, a study in Singapore indicated that the probability of developing anaemia was greater for patients with ESRD, with blood or respiratory disorders (Lau et al., 2015). The difference between the two studies is likely to be accounted for by differences in geographical location, lifestyle, racial and genetic make-up among the study population. In our study, about 1 in ten patients among those who presented with anaemia died during the study period. Similar findings have been reported elsewhere by Herzog et al. (2009) and McClellan et al. (2004). Complications associated with anaemia have been described to an increase in mortality (Silverberg et al., 2009; Lau et al., 2015).

In conclusion, anaemia is prevalent among patients presenting with kidney diseases. Such patients require a thorough evaluation to identify and correct causes of anaemia other than erythropoietin deficiency. Patients with kidney diseases can have anaemia for many reasons, including but not limited to their renal insufficiency. Thus efforts should be placed on the screening the different causes of anaemia among these patients.

\section{Acknowledgements}

Special thanks to Mr. Bakili Shaban, Ms. Happiness Obonyo, Ms. Anna Myovela, Ms. Genia Kibika Ms. Secilia Msafiri, Mr. Musa Semwenda and Mr. Denis Kidera for their support in caring of patients with kidney diseases and proper keeping of data which made this study possible. The authors would like to acknowledge the technical assistance received from Mr. Alphaxard Chibago, Mr Felician Mgassa and Mary Mahuma. 


\section{Authors' contributions}

AJM participated design of the work, collection of clinical data of patients, data analysis and manuscript writing; MYM and MBM participated in data analysis and manuscript writing, JK; collection of clinical data of patients; RT collection of clinical data of patients; Both DPN and IS participated in manuscript writing. All authors have read and approved the final manuscript.

\section{Competing interests}

The authors declare that they have no competing interests.

\section{References}

Coresh, J., Astor, B.C., Greene, T., Eknoyan, G. \& Levey, A.S. (2003) Prevalence of chronic kidney disease and decreased kidney function in the adult US population: Third National Health and Nutrition Examination Survey. American Journal of Kidney Diseases 41: 1-12.

Herzog, C.A., Muster, H.A., Li, S. \& Collins, A.J. (2004) Impact of congestive heart failure, chronic kidney disease, and anemia on survival in the Medicare population. Journal of Cardiac Failure 10: 467-472.

Hill, N.R., Fatoba, S.T., Oke, J.L., Hirst, J.A., O'Callaghan, C.A., Lasserson, D.S. \& Richard Hobbs, F.D. (2016) Global Prevalence of Chronic Kidney Disease - A Systematic Review and Meta-Analysis. PLoS One 11(7): e0158765.

Hsu, C.Y., McCulloch, C.E. \& Curhan, G.C. (2002) Epidemiology of anemia associated with chronic renal insufficiency among adults in the United States: results from the Third National Health and Nutrition Examination Survey. Journal of American Society of Nephrology 13:504-510

Ishimura, E., Nishizawa, Y., Okuno, S., Matsumoto, N., Emoto, M., Inaba, M., Kawagishi, T., Kim, C.W., \& Morii, H. (1998) Diabetes mellitus increases the severity of anemia in non-dialyzed patients with renal failure. Journal of Nephrology 11(2):83-86.

Lau, B.C.V., Ong, K.Y., Yap, C.W., Vathsala, A. \& How, P. (2015) Predictors of anemia in a multi-ethnic chronic kidney disease population: a case control study. SpringerPlus 4:233

McClellan, W., Aronoff, S.L., Bolton, W.K., Hood, S., Lorber, D,L,, Tang, K,L., Tse, T.F., Wasserman, B. \& Leiserowitz, M. (2004) The prevalence of anemia in patients with chronic kidney disease. Current Medical Research and Opinion 20:1501-10.

McFarlane, S.I., Chen, S.C., Whaley-Connell, A.T., Sowers, J.R., Vassalotti, J.A., Salifu, M.O., Li, S., Wang, C., Bakris, G., McCullough, P.A., Collins, A.J., Norris, K.C., Kidney Early Evaluation Program Investigators (2008) Prevalence and associations of anemia of CKD: Kidney early evaluation program (KEEP) and National health and nutrition examination survey (NHANES) 1999-2004. American Journal of Kidney Diseases 51: S46-S55

Mehdi, U. \& Toto, R.D. (2009) Anemia, diabetes, and chronic kidney disease. Diabetes Care 32: 13201326.

Meuwese, C.L., Stenvinkel, P., Dekker, F.W. \& Carrero, J.J. (2011) Monitoring of inflammation in patients on dialysis. Nature Reviews Nephrology 7(3):166-176.

Meyers, A.M. (2015) Significance, definition, classification and risk factors of chronic kidney disease in South Africa. South African Medical Journal 105:233-236.

NCGC (2015) Anaemia Management in Chronic Kidney Disease. NIC Guidelines No. 8. National Clinical Guideline Centre. 
New, J.P., Aung, T., Baker, P.G., Yongsheng, G., Pylypczuk, R., Houghton, J., Rudenski, A., New, R.P., Hegarty, J., Gibson, J.M., O'Donoghue, D.J. \& Buchan, I.E. (2008) The high prevalence of unrecognized anaemia in patients with diabetes and chronic kidney disease: a populationbased study. Diabetic Medicine 25: 564-9.

Otero, A., de Francisco, A., Gayoso, P., García, F., EPIRCE Study Group. (2010) Prevalence of chronic renal disease in Spain: results of the EPIRCE study. Nefrologia 30:78-86.

Peck, R.N., Green, E., Mtabaji, J., Majinge, C., Smart, L.R., Downs, J.A. \& Fitzgerald, D.W. (2013) Hypertension-related diseases as a common cause of hospital mortality in Tanzania: a 3-year prospective study. Journal of Hypertension 31: 1806-1811.

Ramanath, V., Gupta, D., Jain, J., Chaudhary, K. \& Nistala, R. (2012) Anemia and chronic kidney disease: making sense of the recent trials. Reviews on Recent Clinical Trials 7:187-196

Silverberg, D.S., Wexler, D., laina, A. \& Schwartz, D. (2009) The correction of anemia in patients with the combination of chronic kidney disease and congestive heart failure may prevent progression of both conditions. Clinical and Experimental Nephrology 13:101-6.

Smith, R.E. Jr. (2010) The clinical and economic burden of anemia. American Journal of Managing Care 16: S59-66.

Stanifer, J.W., Maro, V., Egger, J., Karia, F., Thielman, N., Turner, E.L., Shimbi, D., Kilaweh, H., Matemu, O. \& Patel, U.D. (2015) The epidemiology of chronic kidney disease in northern Tanzania: a population-based survey. PLoS One 10 (4): e0124506.

Stauffer, M.E. \& Fan, T. (2014) Prevalence of anemia in chronic kidney disease in the United States. PLoS One 9(1): e84943.

Thomas, M., Cooper, M., Tsalamandris, C., Maclsaac, R., Jerums, G. (2005) Anemia with impaired erythropoietin response in diabetic patients. Archives of Internal Medicine 165 (4):466-469.

van Nooten, F.E., Green, J., Brown, R., Finkelstein, F.O. \& Wish, J. (2010) Burden of illness for patients with non-dialysis chronic kidney disease and anemia in the United States: review of the literature. Journal of Medical Economics 13: 241-256.

WHO (2001) Iron deficiency anaemia, assessment, prevention and control: a guide for programme managers 2001. WHO/NHD/01.3 